\title{
Multi-resolution analysis for region of interest extraction in thermographic, nondestructive evaluation
}

\author{
B. Ortiz-Jaramillo ${ }^{a, b}$, H.A. Fandiño-Toro ${ }^{a}$, H.D. Benitez-Restrepo ${ }^{c}$, S.A. Orjuela-Vargas ${ }^{b}$, G. \\ Castellanos-Domínguez ${ }^{a}$ and W. Philips ${ }^{b}$ \\ ${ }^{a}$ Universidad Nacional de Colombia, Cra 27 \# 64-60, Manizales, Colombia; \\ ${ }^{b}$ Gent University, TELIN-IPI-IBBT, St-Pietersnieuwstraat 41, B-9000 Gent, Belgium; \\ ${ }^{c}$ Pontificia Univ. Javeriana, Calle 18 \# 118-250, Cali, Colombia
}

\begin{abstract}
Infrared Non-Destructive Testing (INDT) is known as an effective and rapid method for nondestructive inspection. It can detect a broad range of near-surface structuring flaws in metallic and composite components. Those flaws are modeled as a smooth contour centered at peaks of stored thermal energy, termed Regions of Interest (ROI). Dedicated methodologies must detect the presence of those ROIs. In this paper, we present a methodology for ROI extraction in INDT tasks. The methodology deals with the difficulties due to the non-uniform heating. The non-uniform heating affects low spatial/frequencies and hinders the detection of relevant points in the image.

In this paper, a methodology for ROI extraction in INDT using multi-resolution analysis is proposed, which is robust to ROI low contrast and non-uniform heating. The former methodology includes local correlation, Gaussian scale analysis and local edge detection. In this methodology local correlation between image and Gaussian window provides interest points related to ROIs. We use a Gaussian window because thermal behavior is well modeled by Gaussian smooth contours. Also, the Gaussian scale is used to analyze details in the image using multi-resolution analysis avoiding low contrast, non-uniform heating and selection of the Gaussian window size. Finally, local edge detection is used to provide a good estimation of the boundaries in the ROI. Thus, we provide a methodology for ROI extraction based on multi-resolution analysis that is better or equal compared with the other dedicate algorithms proposed in the state of art.
\end{abstract}

Keywords: Gaussian scale decomposition, infrared nondestructive testing, pulse infrared thermography, region of interest

\section{INTRODUCTION}

The ultimate goal of thermographic inspection in materials is to automatically analyze images providing a pass or fail diagnostic to the operator. In this line of analysis, thermography can detect a broad range of near-surface structuring flaws in metallic and composite components, such as disbonds, delaminations, corrosion and fatigue cracks. These flaws are modeled as a smooth contours centered at peaks of stored thermal energy. The smoothness make difficult the detection of the flaws. ${ }^{1}$ Other difficulty to compose useful interpretations in thermal images is the non-uniform heating of the surface. The non-uniform heating is a phenomena caused by physical features of the inspected object and its external heat sources. Usually, this phenomena is hard to remove because its effect changes from image to image and a simple reference subtraction is unlikely to be convenient. ${ }^{2}$

Previous researches in INDT have faced the same problems explained above. For instance, in a previous research, ${ }^{3}$ we proposed to use multi-resolution analysis and gradient direction for ROI extraction. The methodology uses an a-priori shape in the region growing implying a unavoidable generation of segmentation errors. Generally, all previously considered researches ${ }^{1,3-5}$ requires to chose parameters depending on the object to be inspected. Furthermore, thresholding techniques neglect all the spatial relations among pixels in the image and fails when the image have smooth boundaries which is the case in INDT tasks. ${ }^{6}$ Therefore, this restricts the algorithms

Further author information: (Send correspondence to Benhur Ortiz)

Benhur Ortiz-Jaramillo.: E-mail: bortiz@telin.ugent.be, Telephone: +32 92643412 
to specific applications and fails when they are tested with different materials and defect types. Moreover, the state-of-art in image segmentation shows that the current techniques fail when the image has a background of varying gray level, or when regions vary smoothly in gray level like in INDT tasks.

In this paper, a methodology for ROI extraction in INDT using multi-resolution analysis is proposed, which it is robust to ROI low contrast and non-uniform heating. The former methodology includes local correlation, Gaussian scale analysis and local edge detection. In this methodology local correlation between image and Gaussian window provide interest points related to ROIs. We use a Gaussian window because thermal behavior is well modeled by Gaussian smooth contours. ${ }^{1}$ Also, the Gaussian scale is used to analyze details in the image using multi-resolution analysis avoiding low contrast, non-uniform heating and selection of the Gaussian window size. Finally, local edge detection is used to provide a good estimation of the boundaries in the ROI. Thus, we provide a methodology for ROI extraction based on multi-resolution analysis that is better or equal compared with the other dedicate algorithms proposed in the state of art.

The paper is organized as follows: Section 2 presents scale space representation, homomorphic filtering and the proposed methodology . Afterwards, Section 3 describes the experimental setup, database and the methodologies used for comparison. Section 4 holds the discussion of the results by applying the proposed methodology, and finally Section 5 shows the conclusions of this work.

\section{MATERIALS AND METHODS}

\subsection{Scale space representation}

The scale space representation is an alternative for analyzing signals in multiple levels of resolution. This provides a theory for feature extraction by decomposing the image into pyramid structures. This theory has been developed taking into account the relationship between the signal details and the operators used for the detection of those details. For instance, this kind of representation is very useful when prior information about what resolutions are appropriate for analyzing a signal. ${ }^{7}$ Particularly, the details in INDT applications are represented by smooth contours centered at a peak of stored thermal energy which may represent regions in materials with potential anomalies or failures. Usually, materials under inspection have complex shapes, defects at different depths and different sizes. Then, it is necessary to decompose the image in different scales with the purpose of representing those defects as function of the scale. In such way, it is possible to use a constant operator size for the detection of the potential anomalies. Therefore, we used the scale space theory as an alternative to the analysis of optimal resolutions for feature extraction. In the following the scale space representation, particularly, the dyadic scale space is explained.

Let $X \in \mathbb{R}^{N \times M}$ be an image. Then its multi-scale representation is defined as $X(x, y, j)=G(X(x, y), j) ; j>0$, where $j$ is the scale parameter. Particularly, a popular multi-scale representation is build by using a Gaussian kernel, i.e.,

$$
X(x, y, j)=\sum_{m=-W}^{W} \sum_{n=-W}^{W} w(m, n) X(2 x+m, 2 y+n, j-1),
$$

where $w(m, n)=\sqrt{\frac{1}{2 \pi \sigma^{2}}} \exp \left(-\frac{1}{2 \sigma^{2}}\left(m^{2}+n^{2}\right)\right)$ and $W$ is the size of the Gaussian kernel. Usually, one of the principal issues in the Gaussian scale-space is the selection of the spread parameter $\sigma$, i.e., the sample size between resolution in the scale-space. To cope with this shortcoming, Cong and $\mathrm{De} \mathrm{Ma}^{7}$ proposed a strategy to approximate the scale-space by using a discrete sequence and a particular $\sigma$ by using the Shannon sampling theorem. This method is called dyadic scale-space representation, in which it is proposed a signal decomposition by using a defined $\sigma$, satisfying $\sigma=2^{j}{ }^{7}$ In this work a dyadic scale space is used because it has proved to be a good and efficient discrete representation of the scale-space.

\subsection{Homomorphic filtering}

Homomorphic filtering is a generalized technique for image enhancement and/or correction. It simultaneously normalizes the brightness across an image and increases contrast. That is, in homomorphic systems, the image formation can be described as a multiplication of two or more function. Therefore, by using this kind of filters it is possible to achieve an increase of the edge strength. Also, this kind of image filtering is often used in infrared imaging applications. ${ }^{8}$ Thus, we use the homomorphic filtering with the purpose of increasing the edge strength 
and thus achieve a better ROI boundary detection. In the following a brief explanation concerning homomorphic filtering is provided.

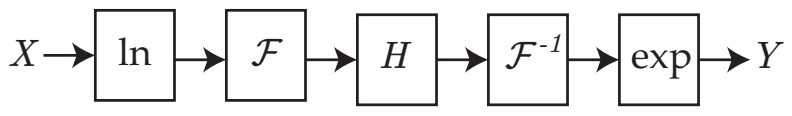

Figure 1. Homomorphic filtering approach for image enhacement.

General procedure for homomorphic filtering is shown in Figure 1. Often an image is expressed as the product of illumination and reflectance components, i.e., $X(x, y)=I(x, y) R(x, y)$. The homomorphic filtering used this representation for introduce a filtering procedure in the Fourier domain. By applying the natural logarithm in both side of the previous product is obtained $\ln (X(x, y))=\ln (I(x, y))+\ln (R(x, y))$. Afterwards, the Fourier transform (FT) is computed with the purpose of applying a filter $H$ in the frequency domain as follows: $F_{X}(u, v)=H(u, v) F_{I}(u, v)+H(u, v) F_{R}(u, v)$, where $F_{I}(u, v)=\mathcal{F}\{\ln (I(x, y))\}$ and $F_{R}(u, v)=\mathcal{F}\{\ln (R(x, y))\}$ are the FT of the illumination and reflectance components of the image, respectively. Then by applying the inverse Fourier transform to $F_{X}$ is obtained $X^{\prime}(x, y)=I^{\prime}(x, y)+R^{\prime}(x, y)$, where $I^{\prime}(x, y)=\mathcal{F}^{-1}\left\{H(u, v) F_{I}(u, v)\right\}$ and $R^{\prime}(x, y)=\mathcal{F}^{-1}\left\{H(u, v) F_{R}(u, v)\right\}$. Afterwards, as the filtering was performed on the logarithm of the image, the inverse operation is taken to obtain the desired enhanced image, i.e., $Y(x, y)=\exp \left(X^{\prime}(x, y)\right)=$ $\exp \left(I^{\prime}(x, y)\right) \exp \left(R^{\prime}(x, y)\right) .^{9}$

Usually, the homomorphic function $H$ is designed by using a high pass Butterworth filter ${ }^{9}$ as follows:

$$
H(u, v)=\frac{1}{1+\left(\frac{D_{0}}{D(u, v)}\right)^{2 n}},
$$

where $n$ and $D_{0}$ are the order of the filter and the cutoff distance from the center, respectively. Then, $D(u, v)$ is defined as follows:

$$
D(u, v)=\sqrt{(u-M / 2)^{2}+(v-N / 2)^{2}},
$$

where $M$ and $N$ are the number of rows and columns of the original image, respectively. ${ }^{9}$

\subsection{Proposed methodology}

In the following the proposed methodology for extracting ROIs in INDT is explained. The proposed methodology is shown in Figure 2. First at all to avoid the scale selection a multi-scale representation of the original image is obtained. Let $X \in \mathbb{R}^{N \times M}$ be an image. Its multi-scale representation is given by an operator $G(X, j)$ such as: $G(X, 0)=X$ and $G(X, j+1)=2 \downarrow h * G(X, j)$, where $2 \downarrow$ and $*$ are downsampling and convolution operators respectively. Afterwards, from each image of the Gaussian scale decomposition is computed the local correlation between a Gaussian window $(g)$ and a local neighbor of size $7 \times 7$. Here, the Gaussian window has a standard deviation equal to one, i.e., $\sigma=1$. As the image was decomposed in multiples scales, it is possible to perform this kind of operation by using a constant window. The local correlation is performed by using convolutions as follows: Let

$$
X_{s t d}=\sqrt{\left(X^{2} * c /(n-1)\right)-\left((X * c)^{2}\right) /(n(n-1))}
$$

be the local standard deviation of a image, where $n=W^{2}$ and $c$ is a matrix of ones of size $W \times W$. Then, the local correlation between the Gaussian window and the image is defined as

$$
X_{\rho}=\frac{(X * g) /(n-1)-(X * c)\left(\sum_{i} \sum_{j} g(i, j)\right) /(n-1)}{X_{s t d} \sigma} .
$$

Then each of the image obtained by the local correlation is interpolated by using bicubic interpolation. Here, the interpolation is performed with the purpose of preserving the original size of the image.

Later, from the set of images obtained by the local correlation and the interpolation procedure it is extracted a set of interest points by using the non-maximal suppression method. The non-maximal suppression method is just a windowing operation in which the pixels in the current neighborhood are set to one if its pixel values are 
equal to the maximum value in that neighborhood and the pixel values are higher than a certain threshold. In this work, the selection of the threshold is set by using a statistical test. Particularly, we used the t-test which test the hypothesis that two attributes are correlated. In this case, it is tested if a gaussian surface match in a neighbor. We did this test under the hypothesis that thermal diffusion is modeled as a smooth contour centered at a peak of stored thermal energy, which may represent regions in materials with potential anomalies or failures. For this reason, the Gaussian model is one of the most used heat flow descriptor since it achieves proper results in approximating heat diffusion processes. ${ }^{10}$

Therefore, the threshold is set as $t=t_{(n-2, \alpha)}$ where $t_{(n-2, \alpha)}$ is a T-statistic with $n-2$ degrees of freedom and p-value equal to $\alpha$. Then, the non-maximal suppression method is performed over the image obtained as $X_{t}(x, y)=X_{\rho}(x, y) \sqrt{\frac{n-2}{1-X_{\rho}^{2}(x, y)}}$. That is, the position $(x, y)$ is a interest point if $X_{t}(x, y)>t$ and $X_{t}(x, y) \geq$ $X_{t}(x+i, y+j)$ for all $i \in[-W, W]$ and $j \in[-W, W]$.

Also, after detecting the interest points the original image $X$ is filtered with a homomorphic filter in the region in which a interest point was detected. This filtering is performed with the purpose of enhancing the edges in the surroundings of the ROI. That is, a local filtering is performed within a circle with center in the interest point and radius equal to $2^{s+1}$, where $s$ is the number of scales in the dyadic Gaussian pyramid. This radius was selected because ROIs cannot be smaller than the minimum scale of analysis. Indeed, if we search for ROIs with constant Gaussian template size and minimum scale of analysis then details in a smaller scales cannot appear in the analysis. Also, the parameters of the filter was selected by using visual inspection to best edge detection $\left(D_{0}=0.5\right.$ and $\left.n=1\right)$.

Finally, edges are extracted from the locally filtered image by using a Canny edge detector. This local edges represents the boundaries of the ROIs. Afterwards, regions within the edge boundaries are filled by using connected components.

\section{EXPERIMENTAL SETUP}

The methodology, used for infrared nondestructive testing, comprises the following stages: a) Feature extraction using Gaussian scale that includes the estimation of interest points by local correlation and statistical hypothesis testing, b) post-processing using homomorphic filtering and local edge detection, and c) Evaluation.

\subsection{Database}

The proposed methodology is tested using three specimens of Carbon Fiber Reinforced Plastic (CFRP) (namely, CFRP006, CFRP007, and CFRP008) having complex shapes, defects at different depths, and with different sizes, as shown in Figures 3(a), 3(b) and 3(c), respectively. Additionally, two other specimens are considered, which are made of PlexiglasTM and aluminium with circular flat bottom holes at different depths, as respectively depicted in Figures 3(d) and 3(e). ${ }^{11}$ Experimental testing is performed by using two flash lamps (Balcar FX 60, 6.4 kJ ), with a $5 \mathrm{~ms}$ pulse, as excitation source. All the thermogram sequences were recorded using a FPA infrared camera (Santa Barbara Focalplane SBF125, 3 to $5 \mu \mathrm{m}$ ), with a $320 \times 256$ pixel array. The number of frames, the frame size, and the sampling period $\Delta t$ of each sequence are listed in Table 1.

Table 1. Parameters used for specimen inspection

\begin{tabular}{lrlr} 
Specimen & Frame number & Frame size & $\Delta t[\mathrm{~ms}]$ \\
\hline CFRP006 & 250 & $220 \times 220$ & 25.2 \\
\hline CFRP007 & 1000 & $292 \times 246$ & 6.3 \\
\hline CFRP008 & 1000 & $284 \times 245$ & 6.3 \\
\hline Plexiglas & 200 & $120 \times 140$ & 2600 \\
\hline aluminium & 1000 & $285 \times 241$ & 6.3
\end{tabular}

\subsection{Evaluation method}

Evaluation of proposed methodology is carried out by using commonly known data mining metrics of misclassification that are described as follows: Let $\hat{B}$ and $B$ be the image with the automatic ROI selection and the ideal ROI selection, where $\mid$ stands for the conditional operator (given that), then, 
- True Positive Rate (TPR): Number of pixels correctly defined as edge pixels, that is, the hits.

$$
T P R=\frac{\hat{B}(x, y) \in \Omega_{p} \mid B(x, y) \in \Omega_{p}}{\operatorname{size}\left\{\Omega_{p}\right\}}, \forall \quad x, y, p
$$

- False Positive Rate (FPR): Number of pixels erroneously defined as edge pixels or false alarms.

$$
F P R=\frac{\hat{B}(x, y) \in \Omega_{p} \mid B(x, y) \notin \Omega_{p}}{\operatorname{size}\left\{\Omega_{p}\right\}}, \forall \quad x, y, p
$$

- Perfect segmentation distance: Euclidean distance between the automatic segmentation and ideal segmentation $(T P R=1$ and $F P R=0)$.

$$
d=\sqrt{F P R^{2}+(1-T P R)^{2}}
$$

- Balanced error: Weighted sum of false positive and false negative rates, i.e.,.: ${ }^{12}$

$$
\varepsilon=\omega_{1} F P R+\omega_{2} F N R,
$$

if assuming $\omega_{1}=\omega_{2}=0.5$, then errors have same contribution. Here,

$$
F N R=\frac{\hat{B}(x, y) \notin \Omega_{p} \mid B(x, y) \in \Omega_{p}}{\operatorname{size}\left\{\Omega_{p}\right\}}, \quad \forall x, y, p
$$

Here, $\Omega_{p}$ and size $\left\{\Omega_{p}\right\}$ are the $p$ th ROI and the number of pixels belonging to the ROI, respectively. Each thermogram sequence is segmented manually and the performance is measured by using the discrepancy, which is based on the number of missegmented pixels,${ }^{1}$ by using the metrics introduced above in each image sequence. The proposed methodology is evaluated by using the following frames of the database: CFRP006 within interval $75.6 \mathrm{~ms}$ to $1260 \mathrm{~ms}$ after heating had started, CFRP007 (81.9 to $630 \mathrm{~ms}$ ), CFRP008 (189 to $630 \mathrm{~ms}$ ), Plexiglas (10.8 to $169.2 \mathrm{~s}$ ) and Aluminium samples (296.1 to $630 \mathrm{~ms}$ ).

Also, attained values of discrepancy for each plate are compared with outcomes achieved for

- Isodata (iterative technique for threshold selection). ${ }^{13}$

- Multi-modal (histogram shape-based method). ${ }^{14}$

- Watershed segmentation method. ${ }^{15}$

\section{RESULTS AND DISCUSSION}

Attained values of mean and standard deviation $(\mu \pm \sigma)$ of discrepancy for each plate are shown in Table 2. First at all, noteworthy is that the false positive rate in watershed procedure and our proposed methodology is $0 \%$. That is, the methodologies are mainly focused on each ROI, taking advantage of its local information instead of processing every simple pixel by thresholding operations. For instance, the multimodal and isodata algorithms produces a $F P R>10 \%$ in those sample where the sample have complex shapes and defects at different depths and sizes (CFRP006, CFRP007 and CFRP008). These results are product of the pixel value dependency of these algorithms, i.e., when regions with gray level values close to the gray level values of the ROIs are considered by chance as part of the ROI. On the other hand, our methodology only segments regions of interest avoiding the limitations produced by the non-homogeneous heating or plate geometry. In general, global segmentation in this kind of applications can achieve a high $T P R$ because of the areas selected such as ROI are large and considered defects are once again taken by chance as ROI. As a result, the reached values of $T P R$ when using thresholding-based procedures are high compared to the ones achieved by the proposed methodology. However, parameters $d$ and $\varepsilon$ shows high values, meaning that those procedures depend on ROI shape and intensity. The results obtained by the Plexiglas sample, the proposed methodology achieved a distance to perfect segmentation $d$ of $0.16 \%$ and the error $\varepsilon$ is $8 \%$, denoting that it is easier to detect defects when these are shallow holes 
Table 2. Performance of considered algorithms appraised for each plate.

\begin{tabular}{|c|c|c|c|c|c|}
\hline \multirow{5}{*}{ CFRP006 } & & Watershed & Isodata & Multimodal & Proposed \\
\hline & TPR & $0.41 \pm 0.12$ & $0.58 \pm 0.10$ & $0.77 \pm 0.11$ & $0.78 \pm 0.11$ \\
\hline & FPR & $0.00 \pm 0.00$ & $0.10 \pm 0.10$ & $0.25 \pm 0.20$ & $0.00 \pm 0.00$ \\
\hline & $d$ & $0.58 \pm 0.12$ & $0.46 \pm 0.09$ & $0.40 \pm 0.14$ & $0.21 \pm 0.11$ \\
\hline & $\varepsilon$ & $0.29 \pm 0.06$ & $0.26 \pm 0.04$ & $0.25 \pm 0.08$ & $0.11 \pm 0.05$ \\
\hline \multirow{4}{*}{ CFRP007 } & TPR & $0.59 \pm 0.09$ & $0.78 \pm 0.11$ & $0.79 \pm 0.12$ & $0.84 \pm 0.05$ \\
\hline & FPR & $0.00 \pm 0.00$ & $0.34 \pm 0.01$ & $0.37 \pm 0.09$ & $0.00 \pm 0.00$ \\
\hline & $\bar{d}$ & $0.4 \pm 0.09$ & $0.42 \pm 0.05$ & $0.45 \pm 0.08$ & $0.15 \pm 0.05$ \\
\hline & $\varepsilon$ & $0.2 \pm 0.04$ & $0.28 \pm 0.05$ & $0.29 \pm 0.06$ & $0.07 \pm 0.02$ \\
\hline \multirow{4}{*}{ CFRP008 } & TPR & $0.58 \pm 0.11$ & $0.93 \pm 0.07$ & $0.95 \pm 0.05$ & $0.73 \pm 0.08$ \\
\hline & FPR & $0.00 \pm 0.00$ & $0.35 \pm 0.07$ & $0.43 \pm 0.07$ & $0.00 \pm 0.00$ \\
\hline & $d$ & $0.43 \pm 0.11$ & $0.37 \pm 0.06$ & $0.44 \pm 0.06$ & $0.26 \pm 0.08$ \\
\hline & $\varepsilon$ & $0.21 \pm 0.05$ & $0.21 \pm 0.04$ & $0.25 \pm 0.03$ & $0.13 \pm 0.04$ \\
\hline \multirow{4}{*}{ Plexiglas } & TPR & $0.51 \pm 0.06$ & $0.67 \pm 0.12$ & $0.78 \pm 0.16$ & $0.83 \pm 0.06$ \\
\hline & FPR & $0.00 \pm 0.00$ & $0.04 \pm 0.02$ & $0.13 \pm 0.13$ & $0.00 \pm 0.00$ \\
\hline & $d$ & $0.48 \pm 0.06$ & $0.34 \pm 0.12$ & $0.30 \pm 0.14$ & $0.16 \pm 0.06$ \\
\hline & $\varepsilon$ & $0.24 \pm 0.03$ & $0.19 \pm 0.05$ & $0.17 \pm 0.06$ & $0.08 \pm 0.03$ \\
\hline \multirow{4}{*}{ Aluminium } & TPR & $0.13 \pm 0.31$ & $0.33 \pm 0.15$ & $0.36 \pm 0.20$ & $0.73 \pm 0.10$ \\
\hline & FPR & $0.01 \pm 0.01$ & $0.01 \pm 0.01$ & $0.05 \pm 0.05$ & $0.00 \pm 0.00$ \\
\hline & $d$ & $0.87 \pm 0.29$ & $0.68 \pm 0.15$ & $0.66 \pm 0.17$ & $0.26 \pm 0.10$ \\
\hline & $\varepsilon$ & $0.44 \pm 0.14$ & $0.34 \pm 0.07$ & $0.33 \pm 0.08$ & $0.13 \pm 0.05$ \\
\hline
\end{tabular}

in isotropic materials. It must be quoted that thresholding-based procedures exhibit a high distance to perfect segmentation for histogram shape based technique.

In case of aluminium sample, due to high thermal conductivity $(209.3 \mathrm{~W} /(K \cdot m))$ in comparison to composite material samples, which are some close to $\left(10^{-5} \mathrm{~W} /(K \cdot \mathrm{m})\right)$, it is difficult to discern whether a hot spot is induced by the defect or by $2 \mathrm{D}$ thermal diffusion. However, attained values of the proposed methodology shows that the methodology is generic enough, at least concerning the change of material composition and defect size and shape.

Generally, based on the above attained results it can be inferred that the proposed methodology is invariant with respect to the inspected material and it may be extended to wider class of materials having low or high thermal conductivity. As a result, the proposed methodology has the best performance in most of the sequences tested.

\section{CONCLUSIONS AND FUTURE WORK}

A methodology to ROI extraction in INDT using local correlation, Gaussian scale analysis and local edge detection was presented. Therefore, the proposed methodology is robust with respect to non-uniform heating because the local correlation (correlation between a gaussian window and a local neighbor) ignores the low spatial frequencies, i.e., the local correlation ignores the components where is located the non-uniform heating. Also, we proved that the defects presented in a material can be modeled by a Gaussian function expressed at different scales. This methodology provides an improvement in region growing compared to thresholding and watershed procedures because is based on local information. Furthermore, we solved a common shortcoming in the nonmaximal suppression method by using as threshold the t-test.

Additionally, the results showed that the proposed methodology is invariant with respect to inspected material and it may be extended to wider class of materials having lower or higher thermal conductivity. As a result, the proposed methodology has the best performance in most of the sequences tested. 
We believe that the combination of multi-resolution analysis, local correlation and statistical tests is useful in other contexts. For example the extraction of regions of interest from infrared images sequences obtained in uncontrolled environments with variable illumination and weather conditions.

\section{ACKNOWLEDGMENTS}

This paper was supported by the project with code 1119-425-20795 of COLCIENCIAS. The authors also wish thanks to Natural Science and Engineering Research Council of Canada (NSERC), the Canada Research Program (CRC): Multipolar Infrared Vision Canada Research Chair (MiViM) for permission to use the databases in active inspection of materials and the program COLCIENCIAS-CIAM (Inter-American Materials Collaboration) 2008.

\section{REFERENCES}

[1] R. Heriansyah and S. A. R. Abu-Bakar, "Defect detection in thermal image for nondestructive evaluation of petrochemical equipments," NDT and E International 42, pp. 729-740, 2009.

[2] Y. A. Plotnikov, N. Rajic, and W. P. Winfree, "Means of eliminating background effects for defect detection and visualization in infrared thermography," Optical Engineering 39, pp. 879-884, 2000.

[3] B. Ortiz-Jaramillo, H. Benitez-Restrepo, J. Garcia-lvarez, and G. Castellanos-Dominguez, "Region of interest extraction based on multiresolution analysis for infrared nondestructive testing," in Proceedings of the 10th International Conference on Quantitative InfraRed Thermography, pp. 417-424, 2010.

[4] I. Abdel-Qader, S. Yohali, O. Abudayyeh, and S. Yehiab, "Segmentation of thermal images for nondestructive evaluation of bridge decks," NDT and E International 41, pp. 395-405, 2008.

[5] R. Usamentiaga, D. F. Garca, and J. Molleda, "Automatic tuning for the segmentation of infrared images considering uncertain ground truth," Electronic Imaging 18, p. 013001, 2009.

[6] A. Thakur and R. S. Anand, "A local statistics based region growing segmentation method for ultrasound medical images," International Journal of Information and Comunications Engineering 1, pp. 141-146, 2005.

[7] G. Cong and S. D. Ma, "Dyadic scale space," in Proceedings of the 13th International Conference on Pattern Recognition, pp. 399-402, 1996.

[8] P. Goyal, "Review of infrared signal processing algorithms," International Journal of Computer Science and Technology 2, pp. 176-180, 2011.

[9] R. C. Gonzales and R. E. Woods, Digital Image Processing, Prentice Hall, Upper Saddle River, New Jersey, USA, 2002 (2nd edition).

[10] R. Fassani and O. Trevisan, "Analytical modeling of multipass welding process with distributed heat source," Brazilian Society of Mechanical Sciences and Engineering 25, pp. 302-305, 2003.

[11] H. D. Bentez, H. Loaiza, E. Caicedo, C. Ibarra-Castanedo, A. H. Bendada, and X. Maldague, "Defect characterization in infrared non-destructive testing with learning machines," NDT and E International 42, pp. 630-643, 2009.

[12] D. Feng, S. Wenkang, C. Liangzhou, D. Yong, and Z. Zhenfu, "Infrared image segmentation with 2-d maximum entropy method based on particle swarm optimization (pso)," Pattern Recognition Letters 26, pp. 597-603, 2005.

[13] R. Dianat and S. Kasaei, "On automatic threshold selection in regression method for change detection in remote sensing images." in Proceedings of the 4th International Symposium on Telecommunications, pp. 1-6, 2008.

[14] M. Sezgin and B. Sankur, "Survey over image thresholding techniques and quantitative performance evaluation," Journal of Electronic Imaging 13, p. 146165, 2004.

[15] L. Vincent and P. Soille, "Watersheds in digital spaces: An efficient algorithm based on immersion simulations," IEEE Transactions on Pattern Analysis and Machine Intelligence 6, pp. 583-598, 1991. 


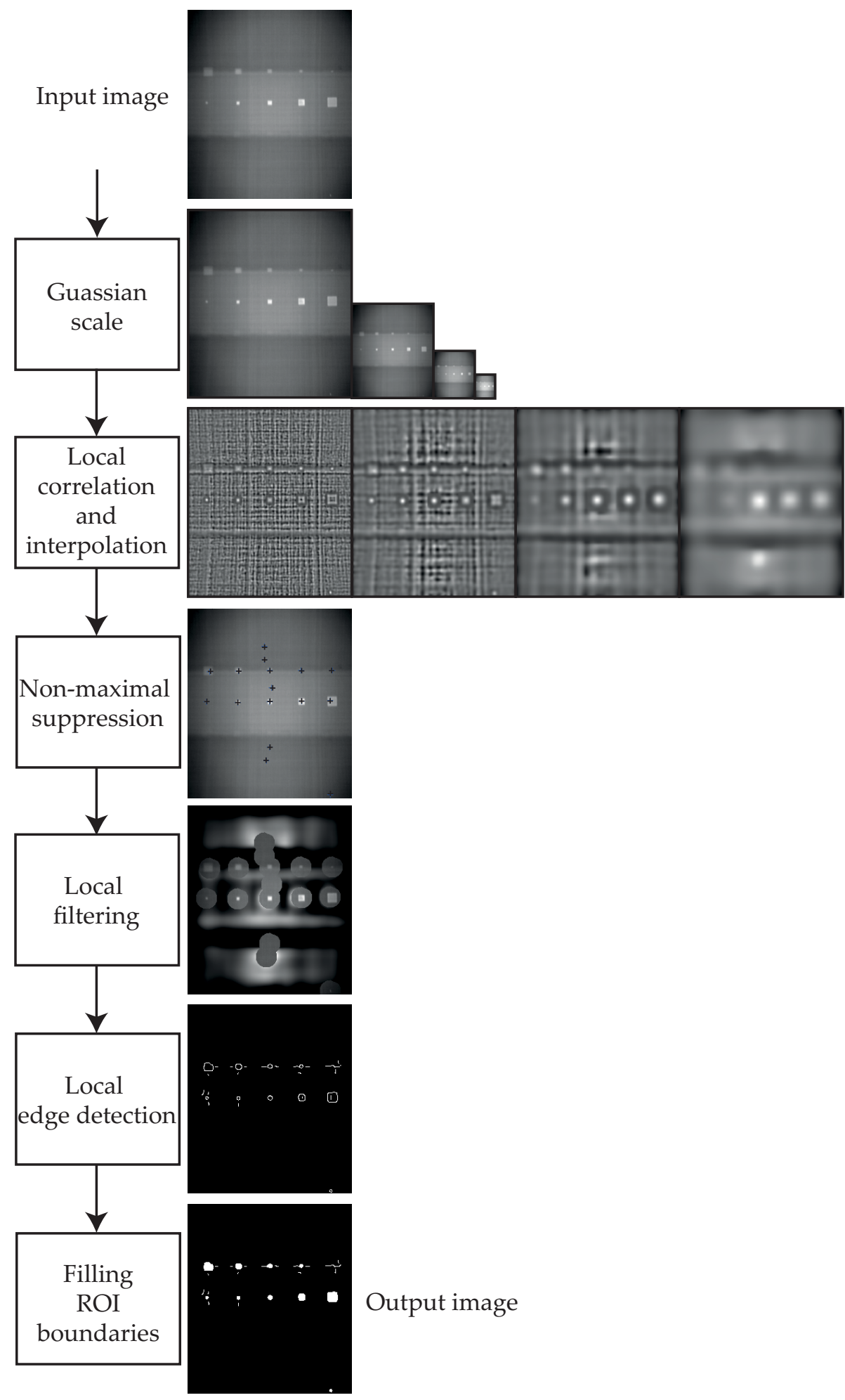

Figure 2. Proposed ROI extraction. Here, the image under test is a frame from the CFRP008 physical sample. 


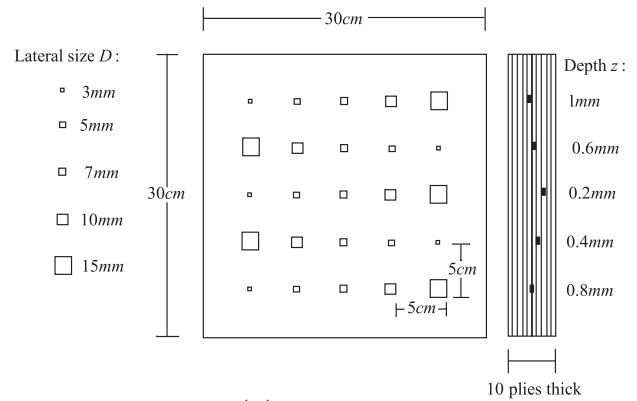

(a) CFRP006

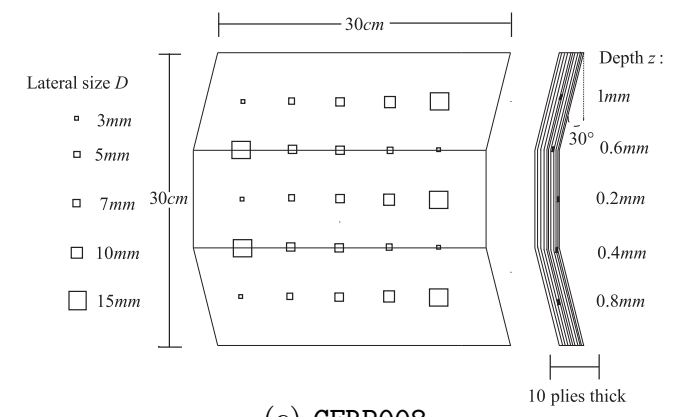

(c) CFRP008

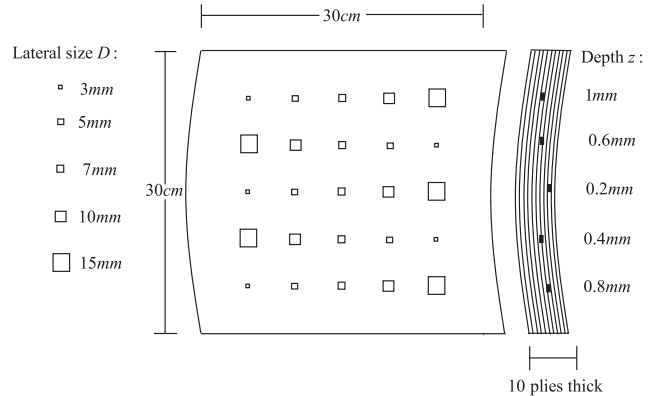

(b) CFRP007

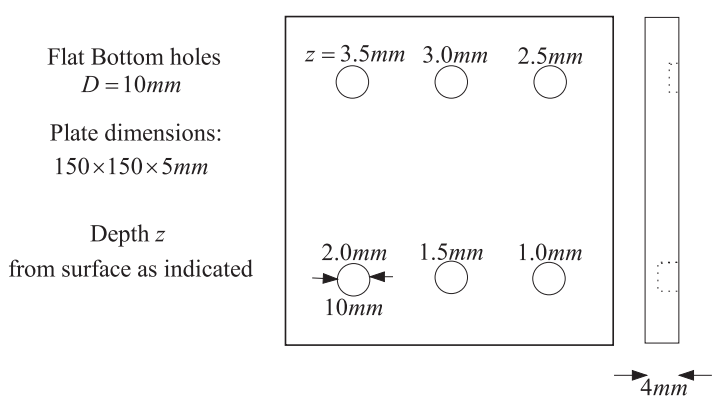

(d) Plexiglas

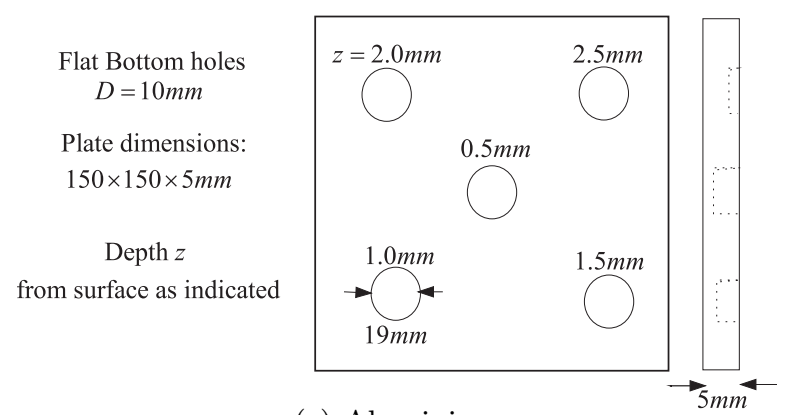

(e) Aluminium

Figure 3. Specification of the inspected plates, taken from. ${ }^{11}$ 